\title{
Phenotype/genotype sequence complementarity and prebiotic replicator coexistence in the metabolically coupled replicator system
}

\author{
Balázs Könnyü ${ }^{1}$ and Tamás Czárán ${ }^{2 *}$
}

\begin{abstract}
Background: RNA or RNA-like polymers are the most likely candidates for having played the lead roles on the stage of the origin of life. RNA is known to feature two of the three essential functions of living entities (metabolism, heredity and membrane): it is capable of unlimited heredity and it has a proven capacity for catalysing very different chemical reactions which may form simple metabolic networks. The Metabolically Coupled Replicator System is a class of simulation models built on these two functions to show that an RNA World scenario for the origin of life is ecologically feasible, provided that it is played on mineral surfaces. The fact that RNA templates and their copies are of complementary base sequences has an obvious dynamical relevance: complementary strains may have very different structures and, consequently, functions - one may specialize for increasing enzymatic activity while the other takes the role of the gene of the enzyme.

Results: Incorporating the functional divergence of template and copy into the Metabolically Coupled Replicator System model framework we show that sequence complementarity 1) does not ruin the coexistence of a set of metabolically cooperating replicators; 2) the replicator system remains resistant to, but also tolerant with its parasites; 3 ) opens the way to the evolutionary differentiation of phenotype and genotype through a primitive version of phenotype amplification.

Conclusions: The functional asymmetry of complementary RNA strains results in a shift of phenotype/genotype (enzyme/gene) proportions in MCRS, favouring a slight genotype dominance. This asymmetry is expected to reverse due to the evolved trade-off of high "gene" replicability and high catalytic activity of the corresponding "enzyme" in expense of its replicability. This trade-off is the first evolutionary step towards the "division of labour" among enzymes and genes, which has concluded in the extreme form of phenotype amplification characteristic of our recent DNA-RNAprotein World.
\end{abstract}

Keywords: Prebiotic evolution, Replicator, RNA World, Coexistence, Complementary RNA strains, Phenotype, Genotype, Template replication

\section{Background}

In a series of simulation studies [1-6] we have shown earlier that a plausible scenario for the prebiotic origins of life can be based on the surface-bound RNA World hypothesis [7-9]. The Metabolically Coupled Replicator System (MCRS) model framework has been developed to demonstrate that a set of different RNA-like macromolecular replicators (ribozymes) cooperating for the

\footnotetext{
* Correspondence: czaran@caesar.elte.hu

${ }^{2}$ MTA-ELTE Theoretical Biology and Evolutionary Ecology Research Group,

Eötvös Loránd University, Pázmány Péter sétány 1/c, Budapest 1117, Hungary

Full list of author information is available at the end of the article
}

production of their own monomers (nucleotides, [10]) can maintain a stable replicator community in which each molecular species (replicator type) catalyses a single reaction of a primitive metabolic reaction network ([1], Figure 1A). The mutualistic interactions among the different catalytic replicator species comprising the MCRS are mediated by their common replication resource the monomers - produced by the replicators themselves through their close chemical cooperation in processing "nutrient" compounds supplied from the environment.

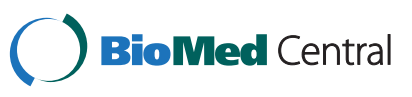

(c) 2014 Konnyu and Czaran; licensee BioMed Central Ltd. This is an Open Access article distributed under the terms of the Creative Commons Attribution License (http://creativecommons.org/licenses/by/4.0), which permits unrestricted use, distribution, and reproduction in any medium, provided the original work is properly credited. The Creative Commons Public Domain Dedication waiver (http://creativecommons.org/publicdomain/zero/1.0/) applies to the data made available in this article, unless otherwise stated. 

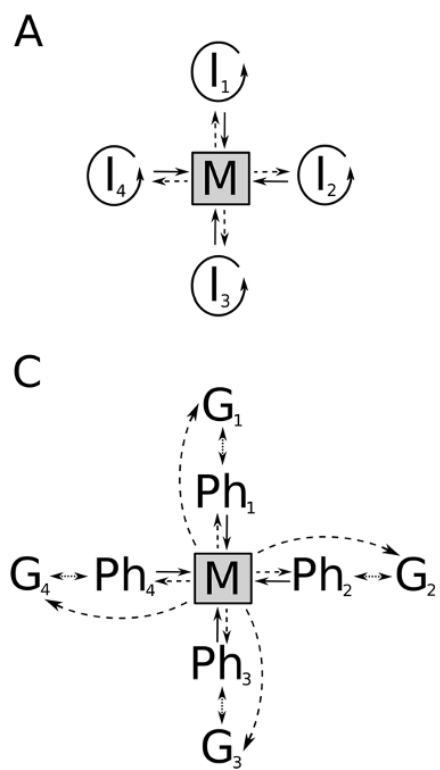

B<smiles>[O-][N+]([O-])(c1ccccc1)c1ccccc1</smiles>

D

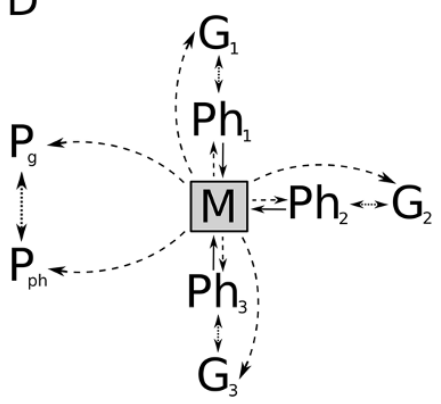

Figure 1 The scheme of the Metabolically Coupled Replicator System without and with template-directed replication. Panel A: The original metabolic replicator system based on [6]. Four autocatalytic metabolic replicators $\left(l_{i}, i=1, \ldots, 4\right.$ within the circular arrows). $M$ is the metabolic reaction network supported by the metabolic replicators as enzymes (solid lines) and producing monomers for their replication (dashed lines). Panel B: the same as Panel A but the system contains a parasitic $(P)$ replicator besides metabolic $\left(l_{i}\right.$ where $\left.i=1 . .3\right)$ ones. Parasites consume monomers produced by the metabolic network but do not contribute to the production of monomers. Pane/ C: the Metabolically Coupled Replicator System in which all reactions of a hypothetical metabolic-network $(M)$ are catalysed (solid arrows) by the phenotype forms of replicators $\left(P h_{x}\right.$, where $\left.x=1, \ldots, 4\right)$. Dashed arrows show that replicators consume the end-products of the metabolic-network (monomers). Dotted, double-headed arrows depict template-dependent replication process which produces phenotype from genotype $\left(G_{x}\right)$ during a replication event, and vice versa. Pane/ $\mathbf{D}$ : shows parasites in the same system like in Panel $\mathbf{C} . P_{g}$ and $P_{p h}$ are the two forms of the parasite.

A working metabolism can be maintained only if each metabolically essential species persists, in spite of the inevitable differences in their overall replicabilities which, in a well-mixed medium, would result in the competitive exclusion of all but the fastest-replicating species and thus in the demise of the metabolic network [1]. Charged mineral surfaces might have offered the first escape for cooperating metabolic replicators from the competitive collapse of their communities [11-13], by anchoring the replicators $[14,15]$ and thus preventing their extensive spatial mixing [1,2]. This simple spatial constraint results in negative frequency dependent feedback regulation: slowly replicating (rare) species enjoy the fitness advantage of a high probability of local metabolic complementation to compensate for their inferior replicabilities. The crucial advantage of rarity comes from the local nature of metabolic interactions on the mineral surface: rare metabolic replicator types have a higher chance to find at least one copy of the more common species within the surface-diffusion range of the metabolites than the common ones to have at least one rare type copy nearby (Additional file 1: Figure S1). This simple spatial regulatory mechanism has been shown to maintain robust coexistence in spite of vast differences assumed in the replicabilities of the different replicator species $[1,6]$.

The original MCRS is a toy model neglecting important physical-chemical details of the supposed RNA World scenario [9]. Some of those details and their consequences for the viability of the metabolic replicator system have been considered in specific modifications of the model during the past decade. Such modifications include the presence of parasites - replicators not contributing to the production but taking part in the consumption of the monomers supplied by cooperating species $([1,6]$, Figure $1 \mathrm{~B}$ and $\mathrm{D})$ - which were shown to persist in the system but generically unable to drive it extinct. Parasites have been proven capable of evolving functions beneficial for the metabolic system, and thus also for themselves: they can acquire new catalytic activities which may improve metabolic efficiency, or they might evolve a slightly better replicase activity than previously available [3], and thus increase the replication rate of the whole replicator community [5].

In the present study we address the dynamical consequences of the fact that the replication of RNA or any known RNA-like macromolecule yields a copy with a monomer sequence different from that of the template - 
a property directly following from nucleotide basepairing rules (A to $\mathrm{U}, \mathrm{C}$ to $\mathrm{G}$ in the case of conventional nucleotide bases). The complementary string of nucleotides in the copy carries the same sequence information as the original (one strain determines the other and vice versa), but its 3D structure, and, consequently, its physicochemical properties - like its enzymatic activity [10] - may be radically different [16]. If the template has an enzymatic activity essential for speeding up a certain reaction step of metabolism, then the copy is almost certainly inactive in that reaction. For the metabolic replicator system as a whole this means that the template is an "enzyme", but the copy is not; it plays the role of a "gene" instead. It is only the "grand-daughter" of a certain template (i.e., a copy of its copy) that has the metabolic activity of the template. Thus, subsequent generations of the same sequence are competitors of each other, besides their obvious genetic relation. It is easy to see that the MCRS model might be very sensitive to this detail: template directed replication is a dynamical issue requiring closer study.

\section{Methods}

The MCRS model with phenotype/genotype distinction

The original (single-sequence) toy model framework (MCRS) has been specified in detail in our previous publications $[1,3,5,6,17]$, therefore we confine model description to the essentials here, with emphasis on new assumptions related to complementary sequence replication.

MCRS is implemented as a stochastic cellular automaton (SCA) model on a square lattice representing the mineral surface to which the replicators are anchored. The topology of the lattice is toroidal (opposite sides merged), to avoid edge effects on the dynamics. Each site may be empty or occupied by a single replicator $i$ at any point of time. Each replicator type has two forms different with respect to their enzymatic activities and complementary sequence-wise: the phenotype form (enzymatically active) and the genotype form (enzymatically inactive, Figure $1 \mathrm{C}$ and D). Thus the number of possible different states for a grid site is $2 n+1$, where $n$ is the number of replicator types (species), i.e., the size of the system.

\section{Basic assumptions of the surface-bound Metabolically Coupled Replicator System}

Each grid site is updated once on average in one generation time using asynchronous random update. The first step of the updating algorithm is the random choice of a grid site, the next state of which (at time $t+1$ ) depends on its own state and those of its neighbours at time $t$. If the focal site is occupied by a replicator, then it becomes empty with a constant probability $p_{d}$. If it is empty, then it may remain so with probability $p_{e}$ (Eq. 1 ), or one of the replicators occupying adjacent sites (from within the replication neighbourhood of the focal empty site, Additional file 1: Figure S1) puts a complementary copy of itself onto the empty site with probability $p_{i}$ (Eq. 2 ).

$$
p_{e}=\frac{C_{e}}{C_{e}+\sum_{j=1}^{r} C_{j}},
$$

and

$$
p_{i}=\frac{C_{i}}{C_{e}+\sum_{j=1}^{r} C_{j}},
$$

where $C_{i}$ and $C_{j}$ are the "claims" of competing replicators to occupy the empty site and $C_{e}$ is the "claim" of the empty site to remain empty (in which case replication does not take place at all). $r$ is the size of replication neighbourhoods (i.e., the maximum number of replicators possibly competing for an empty site). The size of the replication neighbourhood is a parameter of the model: increasing $r$ means that the template may be further away from the empty site on which it claims to place a copy of itself.

The next state of the empty site is determined by a random draw using probabilities $p_{e}$ and $p_{i}$. (Note that $\left.p_{e}+\sum_{i=1}^{r} p_{i}=1\right)$.

The "claim" of a replicator to put a complementary copy of itself to the focal empty site depends on two components: $k_{i}$, a replicator specific constant (replicability), and the local metabolic (monomer) supply $\left(M_{i}\right)$ of replicator $i$ :

$$
C_{i}=k_{i} \cdot M_{i}
$$

\section{Phenotype-genotype difference in catalytic activity}

The local monomer supply $M_{i}$ of replicator $i$ depends on the presence of the enzymatically active phenotype forms of all the metabolic replicator types, each type with at least one copy, within the metabolic neighbourhood of replicator $i$ (Additional file 1: Figure S1). That is, only those replicators can produce daughter copies of themselves which have all the necessary metabolic enzymes for monomer production at their disposal within a small distance - any one of the enzymes missing from the metabolic neighbourhood of replicator $i$ ruins local metabolism and thus excludes the replication of $i$. The formula satisfying these assumptions for the monomer supply $M_{i}$ of replicator $i$ is

$$
M_{j}=\sqrt[n]{\prod_{j=1}^{n} x_{j}^{P h}}
$$

the geometric mean of the copy numbers $\left(x_{j}^{P h}\right)$ of all the different phenotype-forms $j$ within the metabolic neighbourhood of replicator $i: h$ is the size of the metabolic 
neighbourhood (i.e., the number of sites it includes). $M_{i}$ is either zero (if the enzymatically active metabolic replicator set within the metabolic neighbourhood of site $i$ is incomplete), or it is greater than or equal to 1 . Obviously, $M_{i}=0$ implies $C_{i}=0$ (Eq. 3) and, consequently, no chance of replication for the replicator at site $i$.

\section{Phenotype-genotype difference in replicability}

Template-directed replication produces "enzymes" from "genes" and vice versa (Figure $1 \mathrm{C}$ and $\mathrm{D}$ ). These two forms of the same replicator species differ not only in their catalytic activities and monomer sequences. The structural feature that enables the phenotype form ("enzyme") to be catalytically active [10] has an effect on its replicability as well. The compact 3D structure of an efficient "enzyme" form makes it difficult to copy, because it requires more energy and time to unfold during replication than in the case of loosely folded strains. That is, catalytic activity and replicability are expected to be in a trade-off relation in ribozymes [16]. The complementary "gene" form may or may not be easier to copy than the "enzyme" form, but we expect that the "gene" function would be selected for better replicability, implying that the replicability $\left(k_{i p}\right)$ of the "enzyme" form of an evolved replicator species $(i)$ must be smaller than that $\left(k_{i g}\right)$ of the "gene" form of the same species ( $p$ and $g$ in the indexes refer to "phenotype" and "genotype", respectively). Ivica et al. [16] show that about $1.5 \%$ of short ( $35 \mathrm{nt})$ random RNA sequences conform to this assumption, offering a good start for evolution to amplify the difference of phenotype to genotype replicability. The dynamical effect of this trade-off was tested with a comparison of two model versions. In Model I the replicabilities of the "enzyme" and the "gene" were the same, whereas in Model II they were different (with $k_{i p}<k_{i g}$ ).

\section{Parasitic replicators}

Parasitic replicators differ from metabolic ones in that neither of their complementary forms is enzymatically active. That is, both the "phenotype" and the "genotype" of a parasite use monomers produced by the metabolic replicator "phenotypes" for their replication, but neither form contributes to monomer production (Figure 1D).

\section{Replicator mobility}

Replicators bind to the mineral surface reversibly, allowing them some limited mobility in the form of a slow diffusive movement on the surface. This is implemented by using the Toffoli-Margolus algorithm [18] in the model: randomly chosen $2 \times 2$ blocks of sites are rotated $90^{\circ}$ clockwise or anticlockwise with equal (0.5) probabilities. The intensity of replicator diffusion is scaled by the average number $D$ of such sub-lattice rotations per update, so that $D=1$ means four random steps per replicator per generation on average, because one rotation moves four replicators. Note, however, that even with $D=0$ a minimum of replicator mixing is unavoidable, because replicator movement on the surface consists of two independent components: replicative movement $\left(D_{r}\right)$ and diffusive movement $(D)$. The former is an inevitable consequence of the replication mechanism: putting a copy in a site adjacent to that of the template implies the movement of the copy. The relative intensity of replicative to diffusive mixing can be estimated, given that a single Toffoli-Margolus diffusion step $(D=1)$ represents 4 site swaps per replicator per generation, whereas replicative movements result in an average of 0.2 swaps per replicator in each generation (because the death rate of replicators is constant $-p_{d}=0.2-$ a nd replicator densities are stationary). That is, replicative movement corresponds to $D_{r} \cong p_{d} / 4=0.05$ in our model with the parameters above, assuming that the distance between the template and the empty site where the copy is placed is 1 . Then the total mobility of the replicators is $D_{t}=D_{r}+D$, which yields $D_{t}=0.05$ for $D=0$. If the replication neighbourhood is larger than the von Neumann neighbourhood (the four orthogonal neighbours, Additional file 1: Figure $\mathrm{S} 1$ ) of the empty site, then the replicative component of diffusion $\left(D_{r}\right)$ is larger.

\section{Results}

The stochastic cellular automaton consists of 90.000 sites arranged in a $300 \times 300$ rectangular square lattice. All simulations were initiated with $80 \%$ of the sites occupied at random positions by the "genotype" and "phenotype" forms of all replicator types, with both complementary forms of each replicator species represented at equal (10-10\%) proportions.

Replicator coexistence has been shown to critically depend on just a small number of parameters in the original toy model of MCRS [1,6]. The three most effective determinants of coexistence were all related to spatial mixing in that model: the speed of replicator diffusion $(D)$ and the size of replication neighbourhoods $(r)$ both correspond to replicator mobility, whereas metabolic neighbourhood size $(h)$ is the proxy for the average distance that metabolite (and monomer) molecules can cover on the surface before being used in a reaction, degraded or desorbed from the surface. Consequently, $h$ is determined by three factors: the surface diffusibility, the degradation rate and the desorption rate of small metabolite molecules - these three components are lumped in parameter $h$.

We have screened the pheno/geno version of the model for replicator coexistence at different ranges of the same three parameters, keeping all others constant: the death rate of replicators $\left(p_{d}=0.2\right)$, the "claim" of the 
empty site $\left(C_{e}=2.0\right)$ and system size $(n=4)$ were the same in all simulations. Replicabilities were also fixed at $k_{i p}=k_{i g}$ and $k_{p p}=k_{p g}$ in Model I, and $k_{i p}<k_{i g}$ and $k_{p p}<k_{p g}$ in Model II (subscripts $i$ and $p$ denote metabolic replicators and parasite, respectively).

Each simulation produced a quasi-stationary state within the 1.000 generation time frame applied, suggesting that the underlying dynamics may admit attracting fixed point equilibria. The result of a simulation was always one of two possible outcomes: either the whole system survived (with all the metabolic replicator species present), or the replicator community died out altogether. This is just to be expected: the extinction of any one of the metabolically active species stops monomer production and thus kills all the rest, including the parasite if there is one. The survival constraint does not apply to parasites, of course: since they perform no essential function for metabolism, they may go extinct while the metabolic replicators persist.
Wherever the system is persistent, its equilibrium state can be fully specified by the stationary densities of the replicator species, and the phenotype to genotype density ratio within each species. Figures 2 and 3 summarize these output data obtained with Model I to demonstrate the effect of gene/enzyme functional complementarity devoid of the replicability difference between the "enzyme" and the "gene" form of the same replicator type, without and with a parasite, respectively; Figures 4 and 5 show the same simulation outcomes for Model II. The data shown on the figures are the averages of five replicate simulations for each parameter setting.

\section{The effect of replicator mobility}

Replicator mobility is clearly advantageous for the coexistence of the metabolic replicator community in general: increasing any one or both of the diffusion parameters $(D$ and $D_{r}$ ) and the size of the replication neighbourhood $(r)$

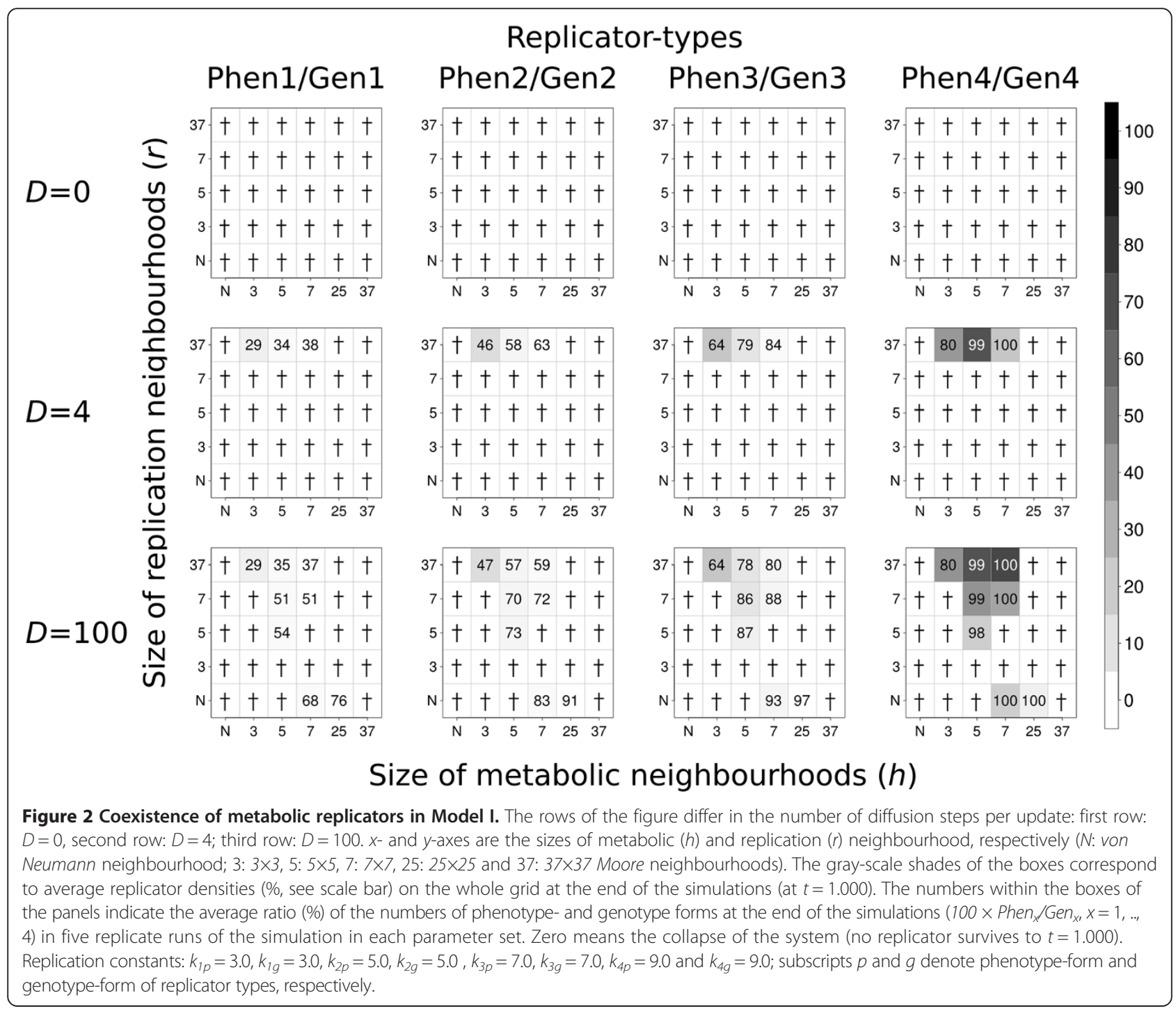


has a positive effect on the persistence of the system, as well as on the average density of replicators on the surface. This conclusion is in line with our previous results [6], applies to Model I and II alike, and it is irrespective of the presence or absence of a metabolic parasite (Figures 2, 3, 4 and 5). These two parameters are almost interchangeable in terms of their direct effects: larger values of any one of them represent more mixing of replicators on the surface. This is obvious for the diffusion parameter, and easy to see for the replication neighbourhood too, because larger $r$ means more distance between template and copy, so that with the replication rate left unchanged, the step length of a single diffusive move increases and thus replicative diffusion $\left(D_{r}\right)$ becomes more intensive with $r$ larger.

There are a few conspicuous exceptions to the trend of monotonous increase in persistence and average replicator density with replicator mobility: at intermediate values of the replication neighbourhood size $(r)$ the system goes extinct in some cases, even though at small and high $r$ it is persistent (cf. Figures 2, 3, 4 and 5). We shall attempt to give a feasible explanation to this seemingly anomalous behaviour of the model in the Discussion.

\section{The effect of metabolite mobility}

The average distance that a metabolite molecule travels on the surface before it becomes the substrate of a metabolic reaction or disappears from the system by desorption or decay is implicit in the size of the metabolic neighbourhood $(h)$. The larger it is the longer the distance within which the products of metabolism - monomers - are available for replicators being copied. In accordance with the conclusions of all previous versions of the MCRS models we find that the pheno/geno version also admits replicator coexistence within an optimum range of 
metabolic neighbourhood size: very small and very large $h$ values are deleterious for system persistence, for essentially the same reasons as in the previous models $[1,3,5,6,17]$. Very small $h$ mimics the fast desorption or decay of metabolites from the mineral surface, so that most of the metabolite molecules cannot be converted to monomers before they disappear from the system. It is difficult (or, in extreme cases, even impossible) to fit a complete set of metabolic replicators into a very small metabolic neighbourhood, therefore it slows down or stops replication everywhere, for lack of monomers.

On the other hand, too large $h$ reduces the advantage of rarity, because rare replicator types are available in the (large) metabolic neighbourhoods of most other replicator types which are capable of faster replication, and thus the more common types outcompete the rare type and the system collapses. In other words, increasing $h$ shifts the system towards the mean-field situation in which it has been shown to go extinct invariably [1] That is, increasing the size of the metabolic neighbourhood increases the chance of metabolic complementation while decreases the advantage of rarity. These counteracting effects are reflected in the optimal intermediate range of $h$ for which coexistence occurs in the simulations (Figures 2, 3, 4 and 5).

\section{The effects of phenotype/genotype functional complementarity}

Comparing the results of the current model (Figures 2, 3,4 and 5) to those of a simulation study that differs from the present one only in the omission of phenotype/ genotype complementarity (Additional file 2: Figure S2) shows that the persistent $(D, r, h)$ parameter range of the pheno/geno version of the MCRS model is somewhat narrower than that of the non-complementary version $[1,6]$. This is to be expected, given that the replicator 


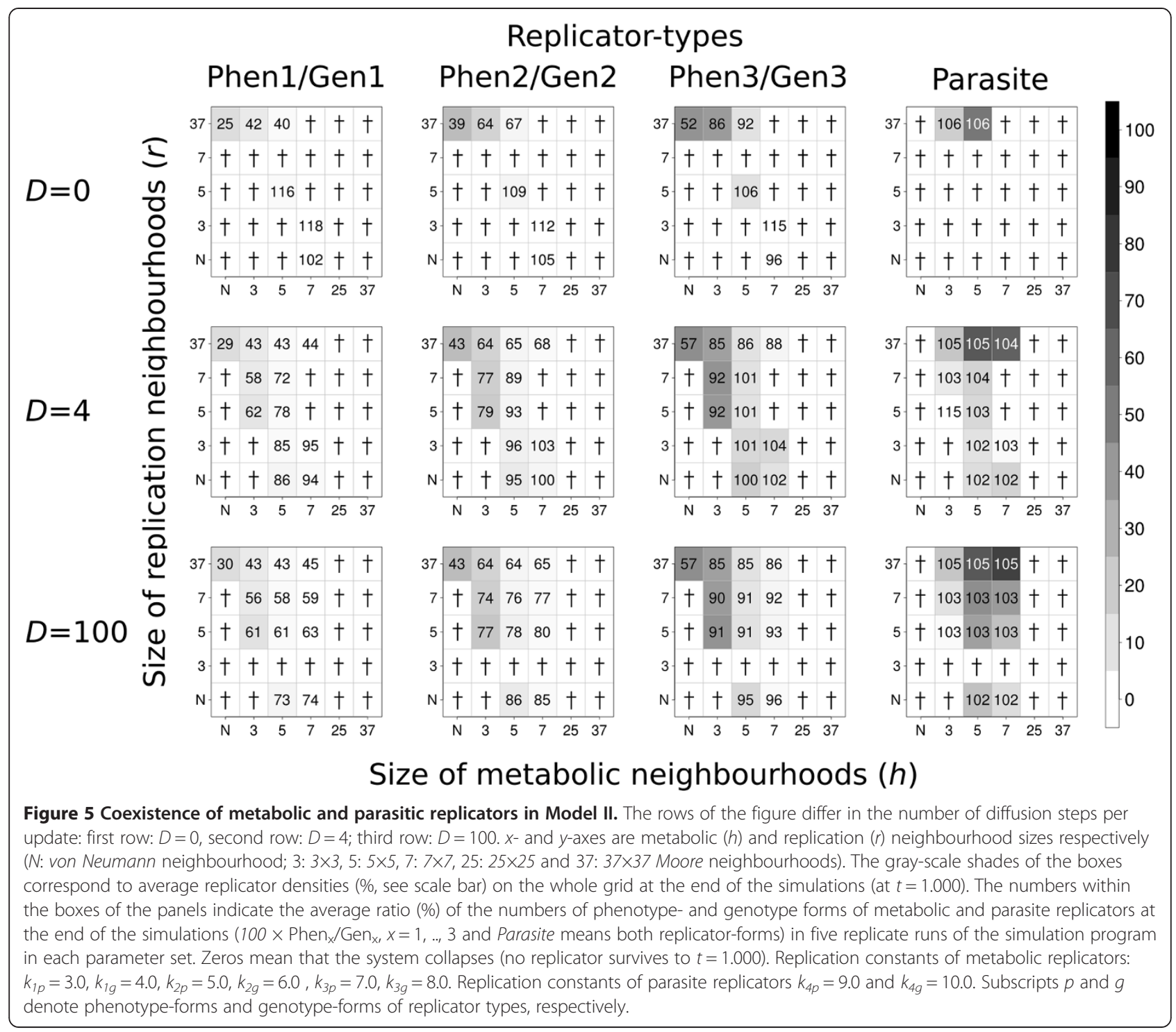

forms with the "gene" function behave somewhat like parasites in the system, using up monomers produced by the enzymatically active forms but not contributing to metabolism directly. The only difference of a "gene" from a parasite is that the copies of a "gene" are functional "enzymes", whereas the replication of parasites yields other parasites with no metabolic functionality. This indirect functional difference is responsible for the persistence of all the "genes" (the "genome") within the system, which does not occur with different parasites - it is only the single fastest replicating parasite that prevails in all versions of the MCRS model, all other parasites are excluded (cf. [6] Figure 3). The presence of the enzymatically inactive "gene" forms dilutes the system in terms of metabolic efficiency, which in turn naturally decreases - but does not destroy - its propensity for coexistence, especially at smaller metabolic neighbourhood sizes.
The effects of the trade-off between gene replicability and enzyme activity

The dynamical consequences of an evolved trade-off between enzyme activity and gene replicability are clearly beneficial for the MCRS: Model I is coexistent in a section of its parameter space that is remarkably smaller than in Model II, and the average density of persistent replicator populations is also smaller everywhere in Model I (compare Figure 2 with Figure 4, and Figure 3 with Figure 5).

\section{The effect of parasites}

The presence of parasites in the pheno/geno model makes little dynamical difference compared to the original MCRS: parasitic replicators remain persistent, sometimes at high densities, but they do not kill the system at any parameter setting studied (Figures 3 and 5). 
Replacing a metabolic replicator with a parasite results in a shift of the coexistent parameter range towards smaller metabolic neighbourhoods, but it is a consequence of the decrease in system size (3 metabolic replicator types instead of 4), not some kind of direct "parasite benefit".

\section{Discussion}

The overall dynamical behaviour exhibited by the pheno/ geno version of the MCRS model is not very different from the single-sequence original $[1,6]$ : metabolically cooperating replicators are coexistent over a large section of the physico-chemically feasible part of the parameter space, and the system is resistant to but, to some extent, also tolerant with its parasites (Figures 2, 3, 4 and 5). Coexistence is due to the general local replication advantage that rare types of metabolic replicators enjoy through a higher probability of metabolic complementation compared to more common replicator types, just like in the single-sequence model. Parasite resistance means that the parasites are not able to kill the system, due to the local metabolic disadvantage of increased parasite density: wherever parasites become abundant, metabolism breaks down and the system - including the many parasites dies out locally, thereby decreasing parasite density. This regulatory mechanism maintains a viable balance of metabolic cooperators and parasites, the latter of which constitute a pool of replicators lacking any essential functionality in the system but always present at considerable density. Parasites are free to mutate and adopt any potentially useful function for MCRS as a whole. The possibilities include the conversion of the parasite to a new metabolic cooperator, a better replicase [3], a ribozyme that contributes to the production of membrane-constituents, or a co-factor of any of these.

\section{Phenotype-genotype complementarity and coexistence} Considering template-copy sequence complementarity (i.e., the phenotype-genotype distinction) does not change this picture, at least not radically. One of the obvious differences is that the coexistence regions of the parameter space, i.e., the parameter settings at which the system is viable, are shifted towards larger metabolic and replication neighbourhoods in the pheno/geno version. This is not very surprising, given that - from a functional point of view - the pheno/geno system is "diluted" by the "gene" forms. Since it is only the "enzyme" form that contributes to metabolism, and each site of the lattice can be home for a single replicator at most, the inevitable presence of the enzymatically inactive "gene" forms scales up effective neighbourhood sizes in inverse proportion to the relative frequency of "enzyme" forms.

A less obvious feature of the pheno/geno simulations is the non-monotone increase of persistence with increasing replication neighbourhood size $(r)$. It shows up consistently on each of Figures 2, 3, 4 and 5. Very small replication neighbourhoods (with $r=4$, i.e., at the von Neumann neighbourhood) seem to be advantageous for coexistence, just as large ones are. But in most cases there exists an intermediate section of the replication neighbourhood scale at which system extinction occurs. Since the persistence of the replicator system depends on the survival of the least abundant metabolic replicator species, in fact the question is: why does a very small replication neighbourhood favour the survival of the least fit (i.e., of smallest replicability) species compared to the others? The answer probably lies in the strong stochastic fluctuation of neighbourhood composition at very small neighbourhood sizes. An empty site with a single replicator in its replication neighbourhood (Additional file 1: Figure S1) has a high chance of being occupied in the next generation by a copy of that single replicator which has no competitor in the same replication neighbourhood (cf. Eqs. 1-4). If a small replication neighbourhood contains a copy of the species of smallest replicability and just a few (or even zero) copies of more common species, then it has a good chance to replicate, much better than it would had it been surrounded by many more replicators from all the fitter species. In other words, larger replication neighbourhoods may include more of the powerful competitors, thus less chance for the least fit species to reproduce. Obviously this kind of advantage of rarity can be exploited only at very small replication neighbourhoods. Larger $r$ decreases this stochastic fluctuation effect, but it also increases spatial mixing due to replication movement. The former effect is deleterious, the latter is beneficial for replicator coexistence, hence the non-monotonous behaviour of system persistence with increasing replication neighbourhood.

All "enzyme" and "gene" forms are distributed on the surface homogeneously in the majority of the parameter settings, except at large metabolic and very small replication neighbourhoods (towards the bottom-right region of the panels in Figures 2, 3, 4 and 5), where the spatial pattern of the system appears patchy with the patches randomly moving across the lattice (see Additional file 3: Movie S1 and Additional file 4: Movie S2). This patchiness, just as the non-monotone behaviour of the system with increasing replication neighbourhood, is the result of another delicate balance of two antagonistic effects. At very small $r$ the system is sparse, so that survival is possible only at locations where the least fit replicator species is present. Each rare type replicator has a "court" of more common species around it, and the patch thus produced is persistent, provided that the system is not mixed too much to drift cooperating replicators far apart (i.e., at small $D$ ) and that the metabolic neighbourhood $h$ is sufficiently large to include the whole patch. Then all the replicators of a patch fit into the same metabolic 
neighbourhood so that they are all supplied by monomers for copying. Very small $r$ guarantees that the rare type survives in spite of $h$ being quite large. Of course, too large metabolic neighbourhoods are fatal for the system because of the shift towards mean-field dynamics leading to system extinction, so the viable range of $h$ at very small $r$ is limited. Note that the sharp borders of the patches dissolve with increasing diffusion, and the pattern of the arena becomes "normal", i.e., nearly homogeneous.

\section{Shifts in "enzyme/phenotype" to "gene/genotype" strain proportions}

The replication of the "gene" form of a certain species yields the "enzyme" form of the same species, but only in the presence of all the metabolically active "enzyme" forms within the metabolic neighbourhood of the strain to be copied. The functional asymmetry of "gene" and "enzyme" forms is reflected in the asymmetry of their copy numbers: the "enzyme" form is always underrepresented in the simulated replicator populations (cf. the proportions given as numbers on Figures 2, 3, 4 and 5). This shift in "enzyme" to "gene" proportions is due to the fact that "enzymes" are always more likely to be copied than "genes", because they are active members of their own metabolic neighbourhoods. Takeuchi and his co-workers come to a similar conclusion with their cellular automata model of an evolving gene-enzyme pair: the gene strand tends to dominate the population [19]. The effect is analogous to the advantage of rarity - we may call it the "advantage of phenotypes" - : since "enzymes" replicate more often and they always yield "genes", it is the latter that become more abundant. This is especially conspicuous in the case of Model I (Figures 2 and 3), where the replicabilities of the "enzyme" and the "gene" forms are the same for each species. We will see in the next section that the consequential loss in system persistence may be compensated by assuming a straightforward trade-off between enzymatic activity and replicability, which in turn may be the result of prebiotic selection for a better "division of labour" between phenotype and genotype strains.

\section{Enzyme activity/replicability trade-off}

Model I differs from Model II in that the latter assumes smaller replicability for the "enzyme" form of a replicator species than for the "gene" form of the same species. The resulting trade-off between enzyme activity and replicability has a few consistent dynamical effects in MCRS: 1 ) the system is persistent in a considerably larger part of the parameter space; 2) wherever the system persists in both models, Model II produces larger overall densities and 3) higher relative frequencies of the phenotype ("enzyme") form (by compensating the advantage of phenotypes, compare Figure 2 to Figure 4 and Figure 3 to Figure 5).

Recall that the enzyme activity part of the trade-off is a physico-chemical necessity: an efficient ribozyme needs to be relatively compact and energetically stable, in order to present the required spatial configuration of critical residues in a steady structure for executing the reaction it catalyses. The replicability part of the tradeoff $\left(k_{p}<k_{g}\right)$ is not so straightforward, because nothing guarantees $a b$ ovo that the complementary strain of a good ribozyme has a loose spatial structure, which would make it easier to copy. To the contrary - the complementary strains of efficient ribozymes have a high chance of being compact themselves too - but this is not a strictly constrained relation either [16]. Therefore, the phenotype/genotype trade-off, the "division of labour" proposed by Ivica et al. [16] between complementary strains of the same replicator species is a possible, but not inevitable feature of the "enzyme"/"gene" pair [20]. The fact that this trade-off is beneficial for the metabolic replicator system as a whole makes it very probable that it will be selected for, so that $k_{p}<k_{g}$ would eventually appear as an evolved feature of the replicator species. This is a conjecture to be studied in detail later, using the MCRS model framework combined with the Vienna algorithm of RNA folding [21]. Regardless of what mechanism the "enzyme"/"gene" trade-off is achieved by, it can be regarded as the first, prebiotic evolutionary step towards a simple form of phenotype amplification (the production of many enzymes from a single gene) - a feature of all recent organisms in which it is attained through transcriptional and translational amplification.

\section{Conclusion}

Incorporating complementary template replication and the consequent phenotype-genotype differentiation into the MCRS framework does not change the basic dynamical properties of the system: all previous conclusions about replicator coexistence and resistance against parasites $[1,6]$ still hold. We do not know - therefore it calls for further studies - whether other theoretical approaches to prebiotic evolution (e.g. SCM - [22], Hypercycle [23]) are also robust against the same modification?

The functional asymmetry of complementary RNA strains is the cause of the shift in phenotype/genotype proportions in MCRS, to the advantage of genotypes. We see the opposite asymmetry in recent organisms: phenotypes (enzymes) are amplified to many copies from just a few copies of their genes. The first step of phenotype amplification might have been the evolutionary acquisition of the enzymatic activity/replicability trade-off which we have shown to be advantageous for the coexistence and the dynamical efficiency of MCRS. More advanced ways of functional differentiation or "division of labour" (including 
the adoption of DNA and the complicated machineries of transcription and translation) might have developed later in some vesicular forms of the metabolic replicator system (see [20]) on the evolutionary route towards the recent DNA-RNA-protein World.

\section{Availability of supporting data}

Additional file 1: Figure S1. introduces the types of neighbourhoods and Additional file 2: Figure S2 shows the results of the original model lacking template complementarity (from [6] Figure 2) Movies show some representative time courses of Model II with the development of heterogeneous replicator patches at different diffusion intensities which either support (Additional file 3: Movie S1 $-D=4$ ) or hamper (Additional file 4: Movie $S 2-D=0$ ) the persistence of parasites.

\section{Additional files}

Additional file 1: Figure S1. Neighbourhoods used in the model. The light grey rectangles depict the Moore (Panel $\boldsymbol{A})$ and the von Neumann (Panel $\boldsymbol{B}$ ) types of neighbourhoods around $X$, which may be a replicator or an empty site depending on the function of the neighbourhood (metabolic or replication neighbourhood).

Additional file 2: Figure S2. Coexistence of metabolic replicators as the function of replicator diffusion $(D)$, metabolic $(h)$ and replication $(r)$ neighbourhood size. The panels of the figure differ in the number of diffusion steps per generation: Panel $\mathbf{A}$ : $D=0$, Panel $\mathbf{B}$ : $D=1$, Panel C: $D=4$ and Panel $\mathbf{D}: D=100 . x$ - and $y$-axes are the sizes of metabolic neighbourhoods $(h)$ and replication neighbourhoods $(r)$ respectively (N: von Neumann neighbourhood; 3: $3 \times 3,5: 5 \times 5,7: 7 \times 7,25: 25 \times 25$ and 37: $37 \times 37$ Moore neighbourhoods). The grayscale shades correspond to average replicator densities (\%) on the whole grid at the end of the simulations (i.e., for $t=1.000$ ). The numbers within panels indicate coexistent/ extinct replicate simulations out of the five repetitions with the same parameter set and different pseudo-random number sequences. From [6].

Additional file 3: Movie S1. Heterogeneous patches on the grid at $D=4$. The spatial dynamics of replicator-types on the lattice from generation 1 to 1000 in Model II. Color shade codes: Light colors: the phenotype ("enzyme") forms; Dark colors: the genotype ("gene") forms of the same replicator type. Green: replicator type 1; Blue: replicator type 2; Red: replicator type 3; Yellow/orange: parasitic replicator type. Other parameters: the size of metabolic neighbourhoods is $h=5 \times 5$ (Mooreneighbourhood), the size of replication neighbourhoods is $r=4$ (von Neumann neighbourhood). Replication constants: $k_{1 p}=3.0, k_{1 g}=4.0, k_{2 p}=5.0, k_{2 g}=6.0$, $k_{3 p}=7.0, k_{3 g}=8.0, k_{4 p}=9.0$ and $k_{4 q}=10.0$; subscripts $p$ and $g$ denote phenotype and genotype forms of replicator types, respectively.

Additional file 4: Movie S2. Heterogeneous patches on the grid at $D=0$. Color codes and parameters as in Additional file 3: Movie S1, except for the replication neighbourhood, which is $5 \times 5$ (Moore).

\section{Competing interests}

The authors declare that they have no competing interests.

\section{Authors' contributions}

BK and TC designed, analysed and interpreted the study. Both authors contributed to writing the manuscript and approved the final version.

\section{Acknowledgements}

The authors acknowledge the financial support of the Hungarian Research Foundation (OTKA) Grant No. K100806. BK acknowledges the financial support of the National Office for Research and Technology (NAP 2005/
KCKHA005). The founders had no role in study design, data collection and analysis, decision to publish, or preparation of the manuscript.

\section{Author details}

${ }^{1}$ Department of Plant Systematics, Ecology and Theoretical Biology, Eötvös Loránd University, Pázmány Péter sétány 1/c, Budapest 1117, Hungary.

${ }^{2}$ MTA-ELTE Theoretical Biology and Evolutionary Ecology Research Group,

Eötvös Loránd University, Pázmány Péter sétány 1/c, Budapest 1117, Hungary.

Received: 4 August 2014 Accepted: 6 November 2014

Published online: 25 November 2014

\section{References}

1. Czárán T, Szathmáry E: Coexistence of replicators in prebiotic evolution. In The Geometry of Ecological Interactions. Edited by Dieckmann U, Law R, Metz JAJ. Cambridge: IIASA and Cambridge University Press; 2000:116-134.

2. Scheuring I, Czárán T, Szabó P, Károlyi G, Toroczkai Z: Spatial models of prebiotic evolution: soup before pizza? Orig Life Evol Biosph 2003, 33:319-355.

3. Könnyű B, Czárán T, Szathmáry E: Prebiotic replicase evolution in a surface-bound metabolic system: parasites as a source of adaptive evolution. BMC Evol Biol 2008, 8:267. doi:10.1186/1471-2148-8-267.

4. Branchiamore S, Gallori E, Szathmáry E, Czárán T: The origin of life: chemical evolution of metabolicsytemin mineral honeycomb? J Mol Biol 2009, 69:458-469.

5. Könnyű B, Czárán T: The evolution of enzyme specificity in the metabolic replicator model of prebiotic evolution. PLOS One 2011, 6:e20931. doi:10.1371/journal.pone.0020931.

6. Könnyű B, Czárán T: Spatial aspects of prebiotic replicator coexistence and community stability in a surface-bound RNA world model. BMC Evol Biol 2013, 13:204. http://www.biomedcentral.com/1471-2148/13/204.

7. Gilbert W: Origin of life: the RNA world. Nature 1986, 319:618.

8. Robertson MP, Joyce GF: The origins of the RNA world. Cold Spring Harb Perspect Biol 2012, 4:1-22.

9. Ruiz-Mirazo K, Briones C, de la Escosura A: Prebiotic systems chemistry: new perspectives for the origins of life. Chem Rev 2014, 114:285-366. doi:10.1021/cr2004844.

10. Chen X, Li N, Ellington AD: Ribozyme catalysis of metabolism in the RNA world. Chem Biodivers 2007, 4:633-655.

11. Copley SD, Smith E, Morowitz HJ: The origin of the RNA world: co-evolution of genes and metabolism. Bioorg Chem 2007, 35:430-443.

12. $\mathrm{Ma}$ W, $\mathrm{Hu}$ J: Computer simulation on the cooperation of functional molecules during the early stages of evolution. PLoS One 2012, 7:e35454 doi:10.1371/journal.pone.0035454.

13. Boerlijst C: Spirals and Spots: Novel Evolutionary Phenomena Through Spatial Self-Structuring. In The Geometry of Ecological Interactions. Edited by Dieckmann U, Law R, Metz JAJ. Cambridge: IIASA and Cambridge University Press; 2000:171-182.

14. Franchi M, Ferris JP, Gallori E: Cations as mediators of the adsorption of nuclec acids on clay surfaces in prebiotic environments. Orig Life Evol Biosph 2003, 33:1-16.

15. Ferris JP: Montmorillonite-catalysed formation of RNA oligomers: the possible role of catalysis in the origins of life. Philos Trans $R$ Soc Lond B Biol Sci 2006, 361:1777-1786.

16. Ivica NA, Obermayer B, Campbell GW, Rajamani S, Gerland U, Chen IA: The paradox of dual roles in the RNA world: resolving the conflict between stable folding and templating ability. J Mol Evol 2013, 77:55-63.

17. Czárán T, Könnyű B, Szathmary E: Metabolically Coupled Replicator Systems: An RNA-World model concept of prebiotic evolution on mineral surfaces. J Sys Chem. in press.

18. Toffoli T, Margolus N: Cellular automata machines: a new environment for modelling. Cambridge: MIT Press, USA; 1987.

19. Takeuchi N, Salazar L, Poole MA, Hogeweg P: The evolution of strand preference in simulated RNA replicators with strand displacement: implications for the origin of transcription. Biology Direct 2008, 3:33.

20. Boza G, Szilágyi A, Kun A, Santos M, Szathmátry E: Evolution of the division of labor between genes and enzymes in the RNA World. PLoS Comp Biol. doi:10.1371/journal.pcbi.1003936. 
21. Lorenz R, Bernhart SH, Höner zu Siederdissen C, Tafer H, Flamm C, Stadler PF, Hofacker IL: ViennaRNA Package 2.0. Algorithms. Mol Biol 2011, 6:26. doi:10.1186/1748-7188-6-26.

22. Szathmáry E, Demeter L: Group selection of early replicators and the origin of life. J Theor Biol 1987, 128:463-486.

23. Eigen M, Schuster P: The hyper cycle. Berlin-Heidelberg-New York: SpringerVerlag; 1979

doi:10.1186/s12862-014-0234-8

Cite this article as: Könnyű and Czárán: Phenotype/genotype sequence

complementarity and prebiotic replicator coexistence in the metabolically coupled replicator system. BMC Evolutionary Biology 2014 14:234.

\section{Submit your next manuscript to BioMed Central and take full advantage of:}

- Convenient online submission

- Thorough peer review

- No space constraints or color figure charges

- Immediate publication on acceptance

- Inclusion in PubMed, CAS, Scopus and Google Scholar

- Research which is freely available for redistribution 